Y. C. Minh and E. F. van Dishoeck, eds.

\title{
Interstellar Matter in Meteorites and Interplanetary Dust
}

\author{
S. Messenger \\ McDonnell Center for Space Sciences and Physics Department, \\ Washington University, St. Louis, MO 63130, USA
}

\begin{abstract}
Meteorites and interplanetary dust particles (IDPs) are primitive solar system materials which contain preserved nebular condensates, circumstellar dust grains and partially preserved molecular cloud matter. The circumstellar dust grains found in meteorites are direct samples of a variety of stars, and provide detailed constraints on models of stellar nucleosynthesis. Hydrogen and nitrogen isotopic anomalies in organic matter in meteorites and IDPs are thought to originate from chemical processes in a presolar molecular cloud. This material is better preserved, but less well characterized among IDPs.
\end{abstract}

\section{Introduction}

Molecular clouds represent a pivotal stage in the evolution of matter in the galaxy. They are largely composed of materials expelled from stars in their last stages of life, either within the massive stellar winds of red giant stars, or from the violent explosions of novae and supernovae. These clouds are also the primary sites of star formation. Virtually all traces of this interstellar heritage have been destroyed in the solar system by extensive chemical and physical reprocessing of materials in the solar nebula and planetary bodies. However, many of the 'minor' planets, asteroids and comets, have escaped significant alteration since the epoch of planet formation 4.6 billion years ago. Meteorites and interplanetary dust particles have provided us with samples of these objects, and their study has led the development in our understanding of the origin and evolution of the Solar System. These primitive bodies have preserved the oldest solar system solids as well as small amounts of pristine stardust and, perhaps, molecular cloud material. The study of these 'presolar' components allows us to directly probe a variety of astrophysical environments which have until recently only been accessible by traditional astronomical techniques.

The isolation of presolar grains in meteorites proved to be an extraordinary challenge in part because there are no chemical or mineral phases known to form in interstellar or circumstellar regions that could not have also formed locally, and thus presolar materials could not be identified on the basis of their chemical compositions alone. However, an important consequence of the thorough chemical and physical processing of solar system materials was its virtually complete isotopic homogenization. That is, the relative abundance of isotopes of any given element is the same throughout the Solar System on scales ranging from micrometers to astronomical units, with typical variations of less than one 
percent. Thus, the one established marker for surviving presolar material is an exotic isotopic composition in at least one element that could not have resulted from processes occurring in the Solar System.

The two types of presolar materials described here - stardust and molecular cloud matter - are isotopically distinct from solar system material, though this results from two very different processes. Circumstellar dust grains are formed from matter freshly synthesized via stellar nucleosynthesis. Their isotopic compositions are determined by the initial mass, chemical composition and age of their parent stars. Isotopic analysis of stardust can thus be used to constrain the probable stellar sources and place stringent limits on nucleosynthetic models. So far, the circumstellar grains found in meteorites are all highly refractory phases which constitute a minor fraction of the interstellar dust population. It is likely that more labile phases have been destroyed by processing in the solar nebula or extensive hydrous and thermal alteration in the meteorite parent bodies. Owing to their small size and the fine grain size of their constituents, stardust has not yet been found in IDPs.

Both IDPs and meteorites also show evidence for the partial preservation of presolar molecular cloud material. In contrast to stardust, molecules formed in molecular clouds obtain their isotopic signatures as a result of chemical processes. Despite the extremely low temperatures of dense molecular clouds $(10-100 \mathrm{~K})$, they are sites of active gas phase and grain surface chemistry. Chemical reactions between neutral molecules at such low temperatures are inhibited by activation energy barriers. However there is no such activation energy barrier if one of the reactants is ionized, and thus 'ion-molecule' reactions are thought to drive the chemical evolution of cold interstellar clouds. Most models of interstellar chemistry are based solely on gas phase ion molecule chemistry (e.g. Millar et al. 1989), although grain-surface reactions are also likely to play an important role (e.g. Tielens 1997).

At such low temperatures the difference in the chemical binding energy between $\mathrm{H}$ and $\mathrm{D}$ can exceed the thermal energy, resulting in extreme mass fractionation during chemical reactions. Consequently, many molecules in cold molecular clouds are observed to have large $\mathrm{D}$ excesses relative to the bulk $\mathrm{H}_{2}$ in these clouds (see Millar \& Hatchell 1998 and references therein). Much smaller effects may also occur for $\mathrm{C}$ and $\mathrm{N}$ at sufficiently low temperatures $(<20 \mathrm{~K}$; Watson 1977; Smith \& Adams 1984; Adams \& Smith 1981). However, the measurement of $\mathrm{C}$ and $\mathrm{N}$ isotopic ratios is a significant observational challenge and so far only marginal detections have been reported (Tielens 1997; Langer \& Graedel 1989; Anderson et al. 1999).

\section{Circumstellar Dust in Meteorites}

The prevailing view for many years was that no presolar matter had survived the (thought to have been) harsh conditions of the solar nebula. This view was supported by the observed isotopic uniformity of meteorites. However, there were several subtle exceptions. Most notably, some primitive meteorites were found to contain isotopically anomalous noble gas components. After years of painstaking work, a research team at the University of Chicago led by Ed Anders succeeded in isolating the carriers of the anomalous gas components by, in 
his words, burning the haystack down to find the needle. The isolation procedure evolved by progressively destroying different phases of the meteorites by harsh acid treatments while tracking the enrichment or loss of the individual gas components. In the end, the noble gas carriers were found to be nanodiamonds (Lewis et al. 1987), SiC (Bernatowicz et al. 1987; Tang \& Anders 1988), and graphite (Amari et al. 1990,1993a). Recently, several additional presolar grain types have been discovered including silicon nitride (Hoppe et al. 1994; Nittler et al. 1995), corundum (Huss et al. 1994; Nittler et al. 1994), and hibonite (Choi et al. 1999). For a more thorough review of circumstellar grains in meteorites, see Anders \& Zinner (1993).

With the exception of nanodiamonds, whose mean size is only $\sim 16 \AA$ (Carey et al. 1987), many individual circumstellar grains are large enough $(\sim 1 \mu \mathrm{m})$ to be measured for their isotopic compositions by secondary ion mass spectrometry (SIMS). Such measurements have shown tremendous variations in the isotopic compositions among individual grains, demonstrating their extrasolar origin. For example, $\mathrm{C}$ isotopic measurements of circumstellar graphite grains have found their ${ }^{12} \mathrm{C} /{ }^{13} \mathrm{C}$ ratios to range from $\sim 2$ to 6000 , far eclipsing the range of values observed among solar system materials, 89 to 93 . The large ranges in isotopic compositions among the grains reflect the fact that they come from diverse stellar sources. In many cases it is possible to measure the isotopic compositions of major and minor elements within the same grain, allowing for better determination of their stellar sources. For example Figure 1 summarizes the $\mathrm{Si}, \mathrm{C}$, and $\mathrm{N}$ isotopic ratios observed among circumstellar $\mathrm{SiC}$ grains.

The abundances of the various presolar grain types range from $\sim 400 \mathrm{ppm}$ for nanodiamonds, to a few ppm for the relatively common graphite and SiC. Corundum, silicon nitride and hibonite are still rarer phases with poorly defined absolute abundances. Despite their low abundances, it has been possible to isolate these presolar grains because of the relatively low background of solar system minerals of these types. The failure, so far, to identify the most abundant type of interstellar grains - silicates - in meteorites may be due to the fact that primitive meteorites are primarily composed of silicates. In this case the challenge is not to find a needle in a haystack, but a particular straw. In a preliminary ion imaging study, Nittler (pers. comm.) has established a rough upper limit on the abundance of presolar silicates in meteorites of $\sim 100 \mathrm{ppm}$. However, it may simply be that presolar silicates have not survived in meteorites, either due to destruction in the nebula or parent body thermal and aqueous alteration.

\section{Evidence for Molecular Cloud Matter in Meteorites}

In addition to the extremely large isotopic anomalies observed among the rare grains of stardust, many primitive meteorites exhibit less pronounced enrichments in $\mathrm{D} / \mathrm{H}$ and ${ }^{15} \mathrm{~N} /{ }^{14} \mathrm{~N}$ ratios in bulk samples. In the past several decades, numerous researchers have subjected primitive chondritic meteorites to a variety of chemical and physical treatments in efforts to isolate the D- and ${ }^{15} \mathrm{~N}$-rich phases. The primary carriers of these anomalies are organic compounds, though D-rich water has also been observed in phyllosilicates in the Semarkona meteorite (Deloule \& Robert 1995). Most meteoritic organic matter is an acid-insoluble 

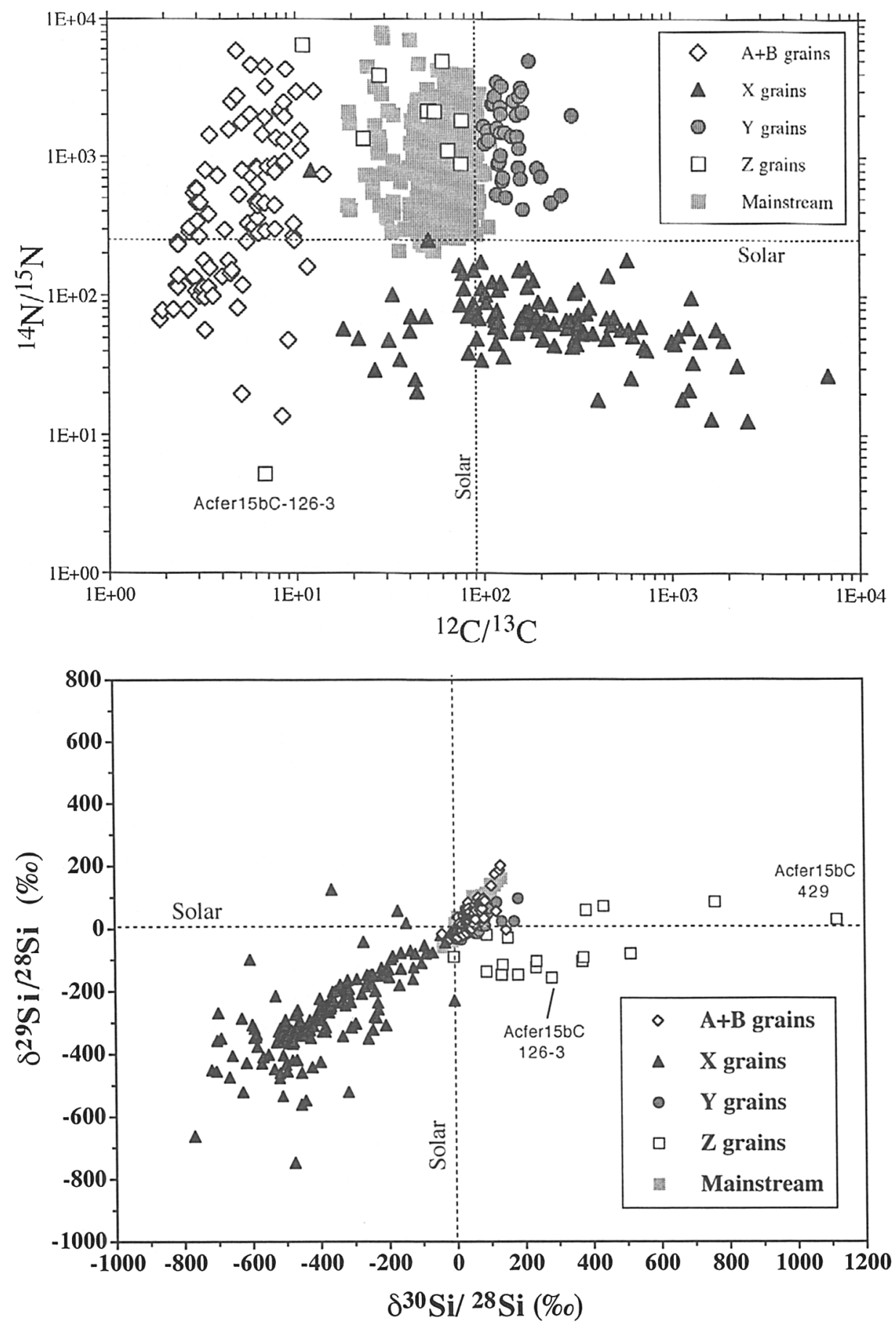

Figure 1. $\mathrm{Si}, \mathrm{C}$, and $\mathrm{N}$ isotopic ratios observed among circumstellar $\mathrm{SiC}$ grains. 
organic polymer similar in structure to terrestrial kerogen. In one detailed study, Kerridge et al. (1987) performed stepwise combustion and pyrolysis experiments on meteoritic kerogen, including $\mathrm{H}, \mathrm{C}$, and $\mathrm{N}$ isotopic measurements for separate temperature steps. Although they were able to identify several isotopically distinct components, the chemical composition of the carriers of these components is unknown, leaving their origin and history unclear.

While less than $10 \%$ of meteoritic organic matter is solvent extractable, more than 400 individual compounds have been identified (see Cronin et al. 1988 for review). Several classes of these compounds have been subject to individual isotopic analysis, including hydroxy, dicarboxylic, and hydroxydicarboxylic acids (Cronin et al. 1993), amino acids (Pizzarello et al. 1991) and aliphatic, aromatic and polar hydrocarbons and monocarboxylic acids (Krishnamurthy et al. 1992). To date, all compounds analyzed exhibit substantial enrichments in $\mathrm{D}$, with the highest $\mathrm{D} / \mathrm{H}$ ratios observed among amino acids, reaching 3.5 times the terrestrial value. The $\mathrm{N}$ isotopic composition of meteoritic kerogen is highly variable among different meteorites, reaching a maximum ${ }^{15} \mathrm{~N} /{ }^{14} \mathrm{~N}$ ratio of 1.25 times the terrestrial value. The solvent extractable organic compounds also generally exhibit excesses in ${ }^{15} \mathrm{~N}$, though the enrichments are somewhat lower than those observed in kerogen (Becker \& Epstein 1982). So far, the highest ${ }^{15} \mathrm{~N}$ enrichment in a well characterized organic phase has been observed in amino acids (Pizzarello et al. 1991).

Deuterium is quickly converted into He in stars, and spallation reactions are far too inefficient to account for the enrichments observed in meteorites. The deuterium excesses must be due to chemical fractionation. Geiss \& Reeves (1981) originally proposed that the origin of the deuterium excesses observed in primitive meteorites was partially preserved molecular cloud material. They argued that at the low temperatures required to reach the level of deuterium fractionation observed in meteorites, chemical reactions would have been prohibitively slow in the solar nebula. While cosmic rays provide a sufficient amount of ionization to drive chemical reactions in molecular clouds, the nebula is thought to have been opaque to ionizing radiation. However, even the highest $\mathrm{D} / \mathrm{H}$ ratios observed in meteorites are far lower than the values observed in interstellar molecules in cold molecular clouds. It is likely that the original complement of molecular cloud material in meteorites has undergone significant chemical processing and mixture with material of solar system origin.

\section{Evidence for Molecular Cloud Material in IDPs}

For the past two decades NASA has routinely collected interplanetary dust particles (IDPs) in the stratosphere. The particles are collected on flat plates coated with viscous silicone oil, mounted on the wings of high altitude research aircraft (Sandford 1987). Particles are removed from the collectors with a micromanipulator and cleansed of the silicone oil prior to further analysis. Most IDPs fall in the size range of 5 to $20 \mu \mathrm{m}$ in diameter. The extraterrestrial origin of IDPs has been established in a number of ways, including the observation of solar flare damage tracks in certain minerals (Bradley et al. 1984), high abundances of solar wind noble gases (Hudson et al. 1981), and the observation of $\mathrm{H}, \mathrm{N}$, and $\mathrm{O}$ isotopic anomalies (Messenger \& Walker 1997 and references therein). 


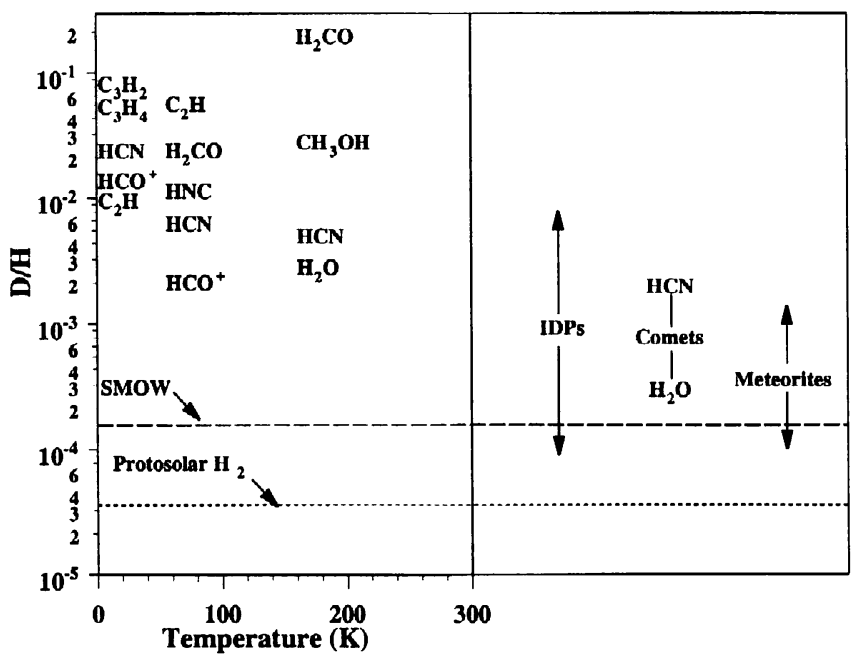

Figure 2. $\quad \mathrm{D} / \mathrm{H}$ ratios observed among primitive solar system materials.

Many IDPs appear to be chemically, mineralogically, and texturally primitive in comparison to meteorites (Bradley et al. 1988). Most IDPs have substantially higher carbon and volatile element (Flynn et al. 1993) abundances than any meteorite. Many IDPs are also extremely fine grained, composed of $\sim 100 \mathrm{~nm}$ subcomponents which display highly unequilibrated mineralogy (see Figure 3 for an image of an IDP). Fine grained IDPs are also commonly highly porous $(\sim 10-50 \%)$ and are thus very structurally weak. Indeed, some disintegrate into numerous pieces upon impact onto the IDP collection surface (these are so-called 'cluster' IDPs). These observations suggest that, unlike the case for meteorites, the material contained in some IDPs has undergone negligible parent body alteration. Consequently, IDPs have long been expected to contain higher abundances, and perhaps a wider array of presolar materials. So far, isotopic measurements of IDPs strongly support this view.

Deuterium and ${ }^{15} \mathrm{~N}$ excesses are common among IDPs, with the largest and most common anomalies occurring among the structurally weak 'cluster' IDPs (Messenger \& Walker 1997 and references therein). Independent isotopic measurements of subfragments of cluster IDPs have further shown the $\mathrm{D} / \mathrm{H}$ ratios to be highly variable, even on a $1 \mu \mathrm{m}$ scale in a given particle. In the most extreme case, the $\mathrm{D} / \mathrm{H}$ ratios of subcomponents of the cluster IDP 'Dragonfly' range from 0.75 to 50 times the terrestrial $\mathrm{D} / \mathrm{H}$ ratio, far exceeding the highest $\mathrm{D} / \mathrm{H}$ ratios observed in any meteoritic sample. The very high deuterium excesses coupled with the micrometer scale isotopic heterogeneity suggest that these particles contain well preserved molecular cloud material. Figure 2 compares the $\mathrm{D} / \mathrm{H}$ ratios observed among primitive solar system materials with direct observations of gas phase molecules in cold molecular clouds. As with $\mathrm{H}, \mathrm{N}$ isotopic ratios also vary considerably within many IDPs, though the maximum observed ${ }^{15} \mathrm{~N} /{ }^{14} \mathrm{~N}$ ratio is only 1.5 times the terrestrial value. No evidence of a $\mathrm{C}$ isotopic anomaly has yet been observed. 


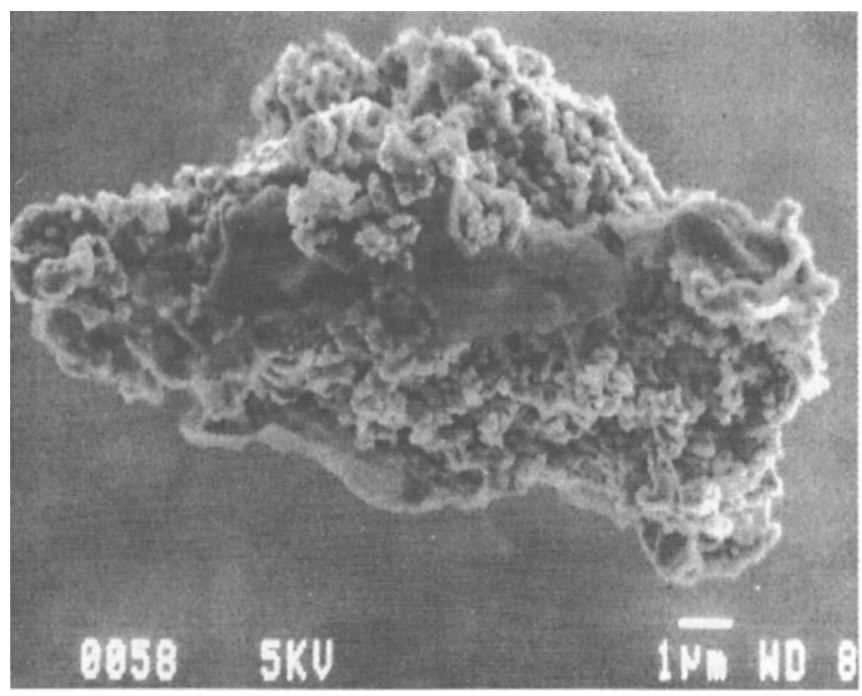

Figure 3. Secondary electron image of an interplanetary dust particle.

Unfortunately, owing to their extremely small sizes ( $\sim 1$ nanogram), very little is known about the nature of the D- and ${ }^{15} \mathrm{~N}$-rich phases. Both anomalies appear to be associated with $\mathrm{C}$, but the degree of $\mathrm{D}$ and ${ }^{15} \mathrm{~N}$ enrichment are not well correlated with each other. It is very likely that there are several isotopically distinct phases distributed unevenly among and within these IDPs.

\section{Interstellar Dust Candidates in IDPs}

Although the large $\mathrm{H}$ and $\mathrm{N}$ isotopic anomalies in IDPs indicate that molecular cloud material is more abundant and better preserved than in meteorites, no circumstellar grains have yet been found in these particles. However, even if stardust is significantly more abundant in IDPs than meteorites it could be difficult to find, given the very low meteoritic abundances and the small amount of material in an IDP (Walker 1994). Given that molecular cloud material and other components in IDPs have apparently escaped significant parent body alteration, it is also possible that circumstellar grain types that were destroyed in meteorites have survived in IDPs. Indeed, there are a number of interesting interstellar grain candidates that have been identified by transmission electron microscopy. Unfortunately, currently available SIMS instruments do not have the sensitivity or spatial resolution necessary to measure the individual (100-300 $\mathrm{nm}$ ) constituents of fine grained IDPs. In one exception to this, McKeegan (1987) observed small oxygen isotopic anomalies $\left({ }^{16} \mathrm{O}\right.$-rich) in several IDPs which contained relatively large refractory minerals. The isotopic effects were not large enough to invoke a presolar origin and are similar to isotopic anomalies observed among refractory mineral components in meteorites. 
Anhydrous, porous IDPs are typically composed of pyroxene grains, $\mathrm{Fe}-\mathrm{Ni}$ sulfides and $100-300 \mathrm{~nm}$ glass nodules, and a variety of less abundant minerals embedded within a carbonaceous matrix. The pyroxene whiskers and platelets are interesting because they appeared to have formed directly from the vapor phase (Bradley et al. 1983), raising the possibility that they formed cither in the solar nebula or a circumstellar outflow. Perhaps the most promising candidates for surviving presolar silicates in IDPs are the common submicrometer glassy objects. Bradley (1994) has suggested that these 'GEMS' (Glass with Embedded Metal and Sulfides) are the 'typical amorphous interstellar silicate' because they share a number of properties that have been inferred from astronomical observations. They are closer in size to the most abundant interstellar grains, and appear to have suffered a high fluence of irradiation, possibly during their residence in the interstellar medium. Recently, Bradley et al. (1999) have shown that the $10 \mu \mathrm{m}$ band from GEMS-rich IDPs provides a good spectral match with those of comets and Herbig Ae/Be stars. Although the evidence is provocative, definitive answers from isotopic measurements have not yet been possible. It is interesting to note however, that these very common components of IDPs are no doubt the best analog material for interstellar silicates.

\section{Concluding Remarks}

Ultimately, a major goal of this work is to use these materials as probes of chemical processes in molecular clouds and the solar nebula, just as the stardust grains have provided direct information on nucleosynthetic processes in stars. Toward that end, several critical questions must be addressed in future work. First, what is the $\mathrm{D} / \mathrm{H}$ ratio of the solid material in interstellar clouds? With the exception of water, none of the gas phase molecules observed in interstellar clouds are likely to be observed in IDPs. Indeed, those molecules represent a trivial fraction of the total deuterium abundance (Tielens 1997), with much of it likely to be incorporated into ices and dust grains. Second, given the common observation of $\mathrm{N}$ isotopic anomalies, why is the $\mathrm{C}$ always isotopically solar? If the $\mathrm{N}$ anomalies do arise from chemical fractionation, the answer is likely to relate to the detailed chemical pathways that lead to the fractionation. Finally, the original arguments posed by Geiss \& Reeves (1981) to justify their assertion that the D excesses arose from interstellar processes may not apply to D-rich IDPs. Aikawa \& Herbst (1999) have recently suggested that there may have been sufficient ionizing radiation present in the outer regions of the solar nebula $(>30 \mathrm{AU})$ to promote ion-molecule chemistry. This is the region of the nebula where short-period comets (which must be the parent bodies of some IDPs) are thought to have formed. At some level, this may be a question of semantics, as the distinction between the cold molecular cloud and the solar nebula becomes poorly defined. The important point is that in order to reach this level of isotopic fractionation, the chemical reactions must have occurred in conditions characteristic of cold molecular clouds. Of course, these issues will all benefit from the identification of the isotopically anomalous components. It is worth noting that even without detailed chemical characterization, the observations of very common $\mathrm{N}$ isotopic anomalies in meteorites and IDPs are well ahead 
of both astronomical observations and interstellar chemical models. This is an important avenue of research that deserves closer attention.

The analysis of stardust in the laboratory has opened up an entirely new branch of astrophysics, providing detailed probes into stellar nucleosynthesis, galactic chemical evolution, and the chemistry of stellar atmospheres. However, we may be reaching a plateau in the types of stardust that can be found in meteorites. The search for stardust in IDPs and, eventually, returned comet samples represents the most promising avenue for identifying surviving interstellar silicates and, perhaps a wide array of other presolar minerals. The instrumental capabilities to make $0.1 \mu \mathrm{m}$ scale isotopic measurements are now at hand with the development of the next generation SIMS instrument (Stadermann et al. 1999). It will shortly be possible to measure the individual components of IDPs in situ, opening up a new window for laboratory astrophysics.

\section{References}

Adams, N.G. \& Smith, D. 1981, ApJ, 247, L123

Aikawa, Y. \& Herbst, E. 1999, ApJ, 526, 314

Amari, S., Anders, E., Virag, A., \& Zinner, E. 1990, Nature, 345, 238

Amari, S., Zinner, E., \& Lewis, R.S. 1993, Meteoritics, 28, 316

Anders, E. \& Zinner E. 1993, Meteoritics, 340, 906

Anderson, I.M., Caselli, P., Haikala, L.K., \& Harju, J. 1999, A\&A, 347, 983

Becker, R.H. \& Epstein, S. 1982, Geochim. Cosmochim. Acta, 46, 97

Bernatowicz, T., Fraundorf, G., Tang, M., Anders, E., Wopenka, B., Zinner, E., \& Fraundorf, P. 1987, Nature, 330, 728

Bernatowicz, T., Amari, S., Zinner, E., \& Lewis, R.S. 1991, ApJ, 373, L73

Bradley, J.P. 1994, Science, 265, 925

Bradley, J.P., Brownlee, D.E., \& Veblen, D. R. 1983, Nature, 310, 473

Bradley, J.P., Brownlee, D.E., \& Fraundorf, P. 1984, Science, 226, 1432

Bradley, J.P., Sandford, S., \& Walker, R.M. 1988, in Meteorites and the Early

Solar System (Tucson: Univ. Arizona Press), 861

Bradley, J.P., et al. 1999, Science, 285, 1716

Carey, W., Zinner, E. Fraundorf, P., \& Lewis, R.S. 1987, Meteoritics, 22, 349

Choi, B.-G., Wasserburg, G.J., \& Huss, G.R. 1999, ApJ, 522, L133

Cronin, J.R., Pizzarello, S., \& Cruikshank, D. 1988, in Meteorites and the Early

Solar System (Tucson: Univ. of Arizona Press), 819

Cronin, J.R., Pizzarello, S., Epstein, S., \& Krishnamurthy, R.V. 1993, Geochim.

Cosmochim. Acta, 57, 4745

Deloule, E. \& Robert, F. 1995, Geochim. Cosmochim. Acta, 59, 4695

Flynn, G.J., Sutton, S.R., \& Bajt, S. 1993, Lunar Planet. Sci., 24, 495

Geiss, J. \& Reeves, H. 1981, ApJ, 340, 906

Hoppe, P., Strebel, R., Eberhardt, P., Amari, S., \& Lewis, R. 1994, Lunar Planet. Sci., 25, 563

Hudson, B., Flynn, G.J., Fraundorf, P., et al. 1981, Science, 211, 383

Kerridge, J.F., Chang, S., \& Shipp, R. 1987, Geo. Cosmochim. Acta, 51, 2527

Krishnamurthy, R.V., Epstein, S., Cronin, J.R., Pizzarello, S., \& Yuen, G.U. 1992, Geochim. Cosmochim. Acta, 56, 4045

Langer, W.D. \& Graedel, T.E. 1989, ApJS, 69, 241

Lewis, R.S., Tang, M., Wacker, J.F., Anders, E.,\& Steel, E. 1987, Nature, 326, 160 
McKeegan, K.D. 1987, Science, 237, 1468

Messenger, S. \& Walker, R.M. 1997, in Astrophysical Implications of the Laboratory Study of Presolar Materials, 545

Millar, T.J. \& Hatchell, J. 1998, Faraday Disc. 109, 15

Millar, T.J., Bennett, A., \& Herbst, E. 1989, ApJ, 340, 906

Nittler, L.R., Alexander, C.M. O'D., Gao, X., et al. 1994, Nature, 370, 443

Pizzarello, S., Krishnamurthy, R.V., Epstein, S., \& Cronin, J. R. 1991, Geochim. Cosmochim. Acta, 55, 905

Sandford, S.A. 1987, Fund. Cosmic Phys., 12, 1

Smith, D. \& Adams, N.G. 1984, ApJ, 284, L13

Stadermann, F.J., Walker, R.M., \& Zinner, E. 1999, Lunar Planet. Sci., 1407

Tang, M. \& Anders, E. 1988, Geochim. Cosmochim. Acta, 52, 1235

Tielens, A.G.G.M. 1997, in Astrophysical Implications of the Laboratory Study of Presolar Materials, 523

Walker, R.M. 1994, in Analysis of Interplanetary Dust, ed. M.E. Zolensky, 203

Watson, W.D. 1977, in CNO Isotopes in Astrophysics, 105

\section{Discussion}

W. M. Irvine: How wide a range of comets are currently available IDPs thought to sample?

S. Messenger: Most IDPs have been in space for thousands of years prior to collection in the stratosphere. Consequently, they have experienced extensive orbital evolution and are no longer associated with their parent comet. It is likely that the IDPs collected sample many comets.

$J$. M. Greenberg: Is there a measure of the relative amount of carbon to silicate in the cluster IDP GEMS?

S. Messenger: The abundance of both carbon and GEMS varies considerably both between different IDPs and within a given particle. However, on average C constitutes roughly $10 \%$ of the mass of IDPs and GEMS-rich IDPs are composed of $\sim 50 \%$ GEMS by mass.

W. A. Schutte: Is there any trace found of the primordial $\mathrm{H}_{2} \mathrm{O}$ in the pre-solar nebula grains inside IDPs, that could have been the source of the Earth's oceans?

$S$. Messenger: The $\mathrm{D} / \mathrm{H}$ ratios observed in IDPs range from below the value of the terrestrial oceans to much higher. On average, the $\mathrm{D} / \mathrm{H}$ ratios of IDPs are significantly higher than the value of ocean water.

T. J. Millar: Have you looked for ${ }^{13} \mathrm{C}$ enrichment in the IDPs?

S. Messenger: The total range of ${ }^{12} \mathrm{C} /{ }^{13} \mathrm{C}$ in IDPs varies by only a few percent. There is no evidence for $\mathrm{C}$ isotopic fractionation in IDPs.

U. G. Jørgensen: Comment. If interstellar grains have a mantle of organic material or ices, this mantle will be destroyed during the chemical extraction procedure in the laboratory (or during the solar system formation). The extracted extrasolar grains are then really stellar dust grain nuclei. It will be important in the future to extract extrasolar grains by physical, non-destructive, methods. 